\title{
An Effective Use of Spectrum Usage Estimation for IEEE 802.22 Networks
}

\author{
Saptarshi Debroy, Shameek Bhattacharjee and Mainak Chatterjee \\ Department of Electrical Engineering \& Computer Science \\ University of Central Florida \\ Orlando, Florida 32816 \\ Email: \{saptarsh, shameek, mainak\}@eecs.ucf.edu
}

\author{
Kevin Kwiat \\ Air Force Research Laboratory \\ Information Directorate \\ Rome, NY 13441 \\ Email: kevin.kwiat@rl.af.mil
}

\begin{abstract}
IEEE 802.22 networks consist of base stations and consumer premise equipments (CPEs) where the base station in each cell opportunistically accesses and allocates (uplink and downlink) channels to all the CPEs in its cell. Information on white space (unused primary channels) availability is reported by the CPEs to the base stations. Thus, a base station's effectiveness to allocate channels are based on its ability to gauge the spectrum usage at various locations.

In this paper, we propose a channel usage estimation framework where a base station uses the spectrum reports from other neighboring base stations to estimate the spectrum usage scenario at any arbitrary location within its cell. Our estimation framework is based on Shepard's interpolation technique for irregular points. We propose a channel allocation scheme that minimizes interference among CPEs and maximizes white space utilization. Through simulation experiments, we demonstrate the accuracy of the estimation technique, utilization of the available spectrum, and efficiency of allocation scheme. We also show that our scheme achieves very low false positives and no false negatives. Finally, we show that the optimal number of base stations that need to be consulted is in accordance with Shepard's bounds ${ }^{1}$.
\end{abstract}

\section{INTRODUCTION}

IEEE 802.22 based wireless regional area network (WRAN) [2] is a cognitive radio technology [3] that operates on the underutilized and unused sub-900 $\mathrm{MHz}$ bands used primarily for TV services. The core components of an IEEE 802.22 network are the base stations (BS) and the consumer premise equipments (CPE). Secondary nodes (BS and CPE) opportunistically access unused or underutilized license bands of primary users. As soon as secondary nodes detect activity from a primary user on a band, they relinquish that band within the channel move time.

Secondary nodes monitor the activity of primary users on a constant basis using spectrum sensing mechanisms, thereby building a knowledge of spectrum usage called the spectrum or channel usage report. This report empowers the secondary nodes to better adapt their transmission parameters to the current conditions. Channel allocation to CPEs is performed by the BS, which is assumed to have an accurate estimation of spectrum usage report of all CPEs in its range. Hence, efficient channel allocation and sustenance of high data rates

\footnotetext{
${ }^{1}$ Approved for Public Release; Distribution Unlimited: 88ABW-20113831, 11JUL11
}

with low interference in WRAN largely rests upon the BS's ability to accurately estimate spectrum usage and allocate unused bandwidth to the CPEs. Though the IEEE 802.22 draft [1] mentions that BSs estimate the spectrum usage at CPE locations by allowing the CPEs to share parameters such as antennae pattern, height, effective isotropic radiated power (EIRP) etc., the actual spectrum usage estimation methodology as well as channel allocation process are unspecified.

Most of the existing work in IEEE 802.22 networks are focused on self-coexistence [6], signal detection or spectrum sensing [8], inter-cell and control channel assignment [4]; however, to the best of our knowledge, the existing literature offers little in terms of investigating spectrum usage estimation in WRANs or using predicted primary occupancy for efficient resource allocation.

In this paper, we propose and evaluate a novel spectrum usage estimation methodology and use it to efficiently allocate data channels to CPEs. We specifically analyze the problem of a BS estimating the spectrum usage at any arbitrary CPE location. To achieve this, the BS opportunistically shares raw spectrum usage reports with selective neighboring BSs and fuses the spectrum data using a modified version of well known Shepard's interpolation technique for irregular data sets in a multi-dimensional space [7]. We extend this estimation framework to an efficient intra-cell channel allocation scheme that minimizes interference among CPEs, maximizes unused channel utilization and increases spatial reuse of such free channels.

We evaluate our framework along three dimensions: accuracy of our proposed estimation technique, utilization of unused channels, and effectiveness of the proposed allocation scheme. We observe that on an average the estimation technique yields $6 \%$ false positive and no false negatives. We also derive bounds on the optimal number of neighboring BSs to be consulted in order to reach a desired level of estimation accuracy. We show how the proposed channel allocation scheme ensures at least $57 \%$ free channel utilization. We also show that in a potentially extreme and highly improbable scenario with all the CPEs in a cell requesting channel allocation at the same time, the proposed scheme successfully allocates $42 \%$ of all the CPEs with a highest possible being $64 \%$. 


\section{INFORMATION GATHERING AND SHARING}

In this section, we define the general model of WRAN. We delineate the sequence of steps taken by a BS and a CPE to establish communication, which facilitates in CPEs providing the BS with spectrum usage reports. We also discuss the methodology by which BSs exchange such reports amongst each other. This information exchange drives our spectrum estimation and allocation in subsequent sections.

\section{A. General WRAN model}

We consider an IEEE 802.22 network that is divided into cells, each having a single BS. A BS can communicate with the CPEs in its cell only through free channels in the spectrum and with its neighboring BSs using pre-defined control channels. The main objective of a BS is to find out free channels in the spectrum and allocate the CPEs a pair of uplink and downlink channels. All the cells are prone to co-channel and adjacent channel interference. The IEEE 802.22 draft [1] requires all the devices in the network to be installed at fixed locations and the BS to be aware of the location of all the CPEs under it.

The challenges of channel allocation in IEEE 802.22 networks in comparison to other conventional networks (cellular) is the absence of pre-defined control channels between BS and CPEs and the very concept that channels to be allocated by the BS are temporally perishable. Therefore a BS has to have two separate units, a cognitive radio enabled unit responsible for sense-transmit-listen and another solely in charge of allocating channels to the CPEs and communicating with other BSs.

\section{B. Sense, transmit and listen}

Initial communication between the $\mathrm{BS}$ and a $\mathrm{CPE}$ takes place through beacon broadcast by the BS and subsequent allocation request by the CPE. A BS employs a sensetransmit-listen approach to facilitate communication. Through its cognitive radio device, the BS tunes itself to a particular channel to find out existence of primary. If free, BS transmits a beacon and listens for a while for allocation requests from CPEs. Details of this sense-transmit-listen policy is governed by the MAC design which is outside the scope of this paper. Only those CPEs can listen to beacons which tune themselves to that particular channel at that time provided that channel is not occupied by the a primary. Therefore, if there are $C$ CPEs in the systems currently looking to get allocated with $K$ total number of channels in the spectrum, then the probability of successful rendezvous between the BS and a CPE is $\frac{C}{K^{2}}$. Although the realistic chances of more than one CPE tuned to the same free channel and responding to a beacon from the BS is still not very well gauged, we adopt a CSMA/CA like approach where the CPEs sense the channel before sending their allocation request to avoid collision.

\section{Opportunistic cooperation among base stations}

The cognitive radio enabled unit of a BS can only tune itself to a particular channel for a stipulated amount of time before moving to the next channel. This motivated us to relieve

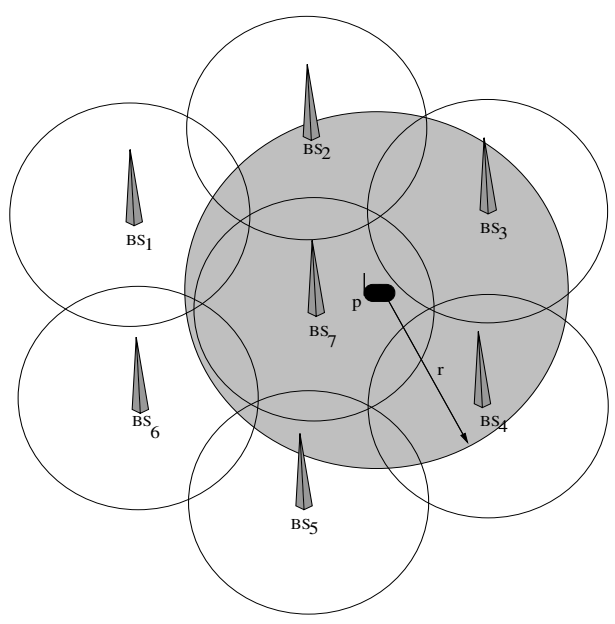

Fig. 1. All the base stations that fall under the circle drawn centering $p$ with search radius $r$

the CPEs from sending their entire spectrum usage reports/ binary decision vectors to the BS because of high payload of such data and increased chances of collision. Instead we allow other BSs within a particular range of the CPE to share their raw spectrum usage reports with the BS in question through their pre-defined control channels. And here we somewhat diverge from conventional cooperative spectrum sensing. In conventional cooperative spectrum sensing, cooperation i.e., sharing of decision vectors (signifying occupancy of channels) among cognitive radio enabled nodes takes place at all times involving all the sensing nodes. Such exhaustive and continuous sharing is unsuitable for an IEEE 802.22 network as it will result unnecessary flooding of spectrum data. Thus we need an on-demand and opportunistic cooperative spectrum sharing technique among selected BSs.

In our proposed scheme. such sharing is triggered by the reception of any channel allocation request and that is when the BS unit responsible for channel allocation takes over. Selection of participating BSs or data points ${ }^{2}$ depends on the search radius specified. Intuitively a bigger search radius will incorporate more BSs resulting in enhanced accuracy of estimation but at a price of increased complexity. More on optimal choice of search radius is discussed in Section III and Section V. It is to be mentioned that the proposed opportunistic spectrum collaboration retains the best of both centralized and distributed cooperation techniques.

Fig. 1 shows an IEEE 802.22 network with seven different cells and each having a single BS. The figure shows $B S_{2}$, $B S_{3}$ and $B S_{4}$ share their spectrum usage with $B S_{7}$ as they come under the sharing range from $p$ within radius $r$.

Once BS successfully receives spectrum usage reports from neighboring BSs, it estimates of spectrum usage scenario at the CPE which we discuss in the next section.

\footnotetext{
${ }^{2}$ We use the cognitive base stations as the data gathering points, hence we also refer to them as 'data points' in future mathematical formulation
} 


\section{Estimation of Spectrum Usage AT CPE}

In this section we discuss the interpolation technique used by the $\mathrm{BS}$ to estimate the spectrum usage at any arbitrary CPE location in the cell.

Estimating the spectrum usage at any arbitrary point from a given set of points is non-trivial. In essence, we seek to define a continuous two-dimensional interpolation function which passes through all the given irregularly-spaced data points.

Let us consider $N$ cognitive radio enabled $\mathrm{BSs}$ and coordinates of the $i$ th $\mathrm{BS}$ be $\left(x_{i}, y_{i}\right)$. Also, this BS records some data value of $z_{i}$. The data value can correspond to one of the many radio parameters like SNR, duty cycle, or detected energy for a particular channel that the BS is sensing. Now, given $N$ such triplets $\left(x_{i}, y_{i}, z_{i}\right)$, we seek to find a two dimensional interpolation function $f(x, y)=z$ that will be continuous and differentiable, passing through all the data points i.e., $f\left(x_{i}, y_{i}\right)=z_{i}$, and conforming to real life values.

We start with a basic approach to interpolate values using weighed averages. Let $e_{i}$ be the value of the detected energy from the spectrum usage report of $B S_{i}$. If $d_{i}$ is the Euclidean distance between $B S_{i}$ and any arbitrary $\mathrm{CPE} c$, then the interpolation function considering all the BSs can be defined as:

$$
F_{1}(c)= \begin{cases}\frac{\sum_{i=1}^{N}\left(d_{i}\right)^{-k} e_{i}}{\sum_{i=1}^{N}\left(d_{i}\right)^{-k}} & \text { if } d_{i} \neq 0 \text { for all } B S_{i}(k>0) \\ e_{i} & \text { if } d_{i}=0 \text { for some } B S_{i}\end{cases}
$$

Although this technique of finding expected value at an arbitrary point is easy to compute, it overlooks some key aspects like the number, locations, and the relative positions of the known data points with respect to point where the value is to be determined. As far as number of data points is concerned it is intuitive that as the number of data points increases, more will be accuracy. However, it is infeasible to use the spectrum value of every BS in the network for estimation of spectrum data at any $\mathrm{CPE}$ in the network regardless of its location. In this regard, we make use of the Shepard's [7] method to find a computationally reasonable interpolation function for irregularly spaced data points in a two dimensional region.

\section{A. Selection of points}

As we mentioned earlier, one of the key issues of successful approximation is to choose nearby data points prudently. We only select those data points which are sufficiently close to a CPE because the effect of those values on that CPE will be more than the distant points.

Let us consider a collection of data points near CPE $c$ with the search radius being $r$. Choice of this search radius determines the number of data points to be selected for interpolation and thus should be chosen to select such data points that have the most influence on point $c$. Let $R_{c}=\left\{B S_{i} \mid d_{i} \leq r\right\}$ be the set all data points within the search radius. where $d_{i}$ is the distance of $B S_{i}$ from CPE $c$. We also define the set $R_{c}^{n}=\left\{B S_{1}, B S_{2}, \ldots \ldots, B S_{n}\right\}$ such that $0 \leq d_{1} \leq d_{2} \leq \ldots \ldots . \leq d_{n}$, which denotes the BSs in an ascending order of their distances from $c$. A weighing function dependent on the search radius is defined as [7]:

$$
p_{i}=\left\{\begin{array}{lr}
\frac{1}{d} & \text { if } 0<d \leq \frac{r}{3} \\
\frac{27}{4 r}\left(\frac{d}{r}-1\right)^{2} & \text { if } \frac{r}{3}<d \leq r \\
0 & \text { if } r<d
\end{array}\right.
$$

Thus considering the effect of distance of the data points, the interpolation function can be modified as:

$$
F_{2}(c)= \begin{cases}\frac{\sum_{B S_{i} \in R_{c}^{n}}\left(p_{i}\right)^{2} e_{i}}{\sum_{B S_{i} \in R_{c}^{n}}\left(p_{i}\right)^{2}} & \text { if } d_{i} \neq 0 \text { for all } B S_{i} \\ e_{i} & \text { if } d_{i}=0 \text { for some } B S_{i}\end{cases}
$$

However, this interpolation function does not reflect the effect of direction of those data points i.e., the relative angle they make with each other. In the following section we will try to incorporate the directional effects.

\section{B. Direction of data points}

We seek to incorporate the directional effect of the data points by considering all the possible set of angles that each data point makes with all other data points. Thus the directional weighting term for each selected data point $B S_{i}$ near $c$ can be defined as

$$
a_{i}=\frac{\sum_{B S_{j} \in R_{c}^{n}}\left(p_{j}\right)\left[1-\cos \angle B S_{i} c B S_{j}\right]}{\sum_{B S_{j} \in R_{c}^{n}}\left(p_{j}\right)} \forall j \neq i
$$

Now, considering all the effects of number, distances, and directions of data points on point $c$, we define the weighing factor as $w_{i}=\left(p_{i}\right)^{2}\left(1+a_{i}\right)$. It is to be noted that in the directional weighing term $a_{i}$, the distance weighting factor $p_{j}$ is included in the numerator and the denominator because points near $c$ should be more important in shadowing than distant points. Thus, the final interpolation function considering the distance and direction factors is:

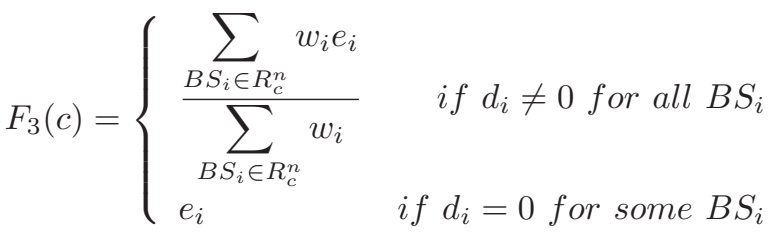

The interpolation function $F_{3}(c)$ meets all the requirements (i.e., defined at every point, continuous and differentiable) which we need to compute the spectrum usage scenario at CPE $c$.

Once the received noise from the primaries is estimated for all the channels, BS predicts the occupancy matrix (binary decision vectors) of $c$ by comparing the estimated value with a predefined threshold. The predicted occupancy matrix determines the potentially available channels at $c$. 


\section{Allocation of Channel among CPES}

In this section we describe the technique that a BS applies to allocate channels to the CPEs.

Once the potentially available channels at $c$ is determined, the BS initiates channel allocation process. Successful channel allocation to a CPE involves allocating a pair of channels, downlink and uplink. Only those channels can be allocated as downlinks and uplinks which are free both at the CPE and at BS, otherwise it will cause harmful interference with primary transmission. We could have provisions for false negatives where BS estimates a channel to be free but actually not, but our extensive simulation results show that the estimation technique is false-negative proof.

Whenever two channels are allocated to a CPE, they are added to the sets of allocated downlink and uplink channels. This helps the BS to ensure spatial reuse of the channels within its cell. The BS is thus responsible to ensure that those channels are only allocated to CPEs which are sufficiently apart to cause co-channel interference. Adjacent-channel interference is also taken care of by BS.

If CPE $c$ wants to relinquish its channels, they are removed from their respective sets. There may be several reasons for channel relinquishment. Session termination by the CPE $c$ or change in spectrum usage scenario in either BS or CPE side because of arrival of primary incumbent. In that case these channels cannot be used any longer until they are freed by primary incumbent, and thus the CPE in question has to be reallocated within the channel move time or kept starving.

Ideal Case: In an ideal scenario of Fig. 2 three CPEs $p, q$ and $r$ reside under $B S_{1}$ and $B S_{2}$. Now it does not matter which channels are allocated to CPEs $q$ and $r$ with respect to $p$ as the former two are under different BSs. Fig. 2 shows that $q$ is given $c h_{3}$ and $c h_{4}$ whereas $r$ is given $c h_{1}$ and $c h_{2}$ which are the same as that of $p$. It is of importance what channels are allocated to CPEs $q$ and $r$ with respect to each other as they reside in the same cell and within their mutual interference region.

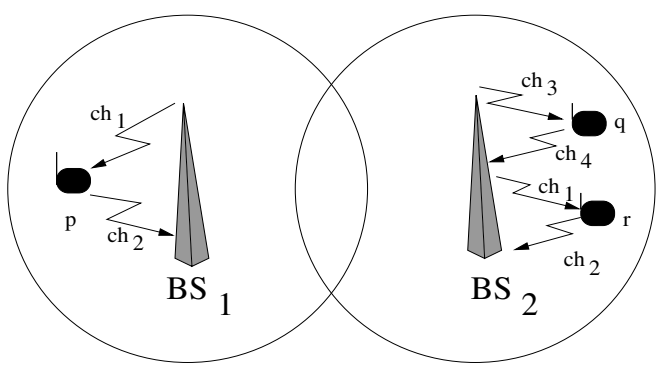

Fig. 2. Channels allocated to $p$ and $q$ are $\left(c h_{1}, c h_{2}\right)$, and $\left(c h_{3}, c h_{4}\right)$ respectively. CPE $r$ under $B S_{2}$ at a considerable distance apart from $p$ is allocated the same channels as $p$.

CPE in cell overlapped region: When a CPE lies in the overlapping region of two or more cells, then it receives beacons from all the corresponding BSs. In such cases, the $\mathrm{CPE}$ can only respond to any one of the BSs. Fig 3 describes such a situation where CPE $p$ lies in the overlapping region of the cells of $B S_{1}$ and $B S_{2} . p$ chooses to associate with $B S_{1}$

and sends a channel allocation request. Channel allocation to node $p$ by $B S_{1}$ takes place in the same technique discussed. Fig. 3 describes the steps taken by $B S_{1}$ to allocate CPE $p$ channels $c h_{1}$ and $c h_{2}$ as uplink and downlink respectively. It is to be noted that the response from node $p$ will also reach $B S_{2}$, but $B S_{2}$ will ignore that as the response message from $p$ carries the unique identifier of $B S_{1}$ and not of $B S_{2}$.

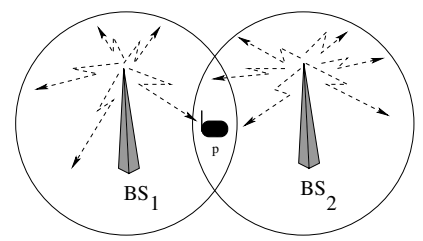

(a) Node $p$ receives beacons from both BSs.

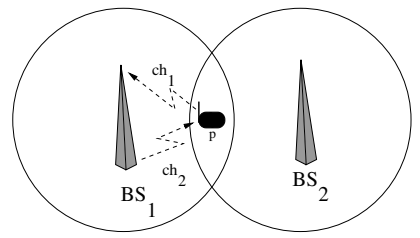

(c) $B S_{1}$ allocates uplink and downlink to node $p$

Fig. 3. A case where $p$ resides in the overlapping region of cell $i$ and $j$

\section{Simulation MOdel AND RESUlts}

We conduct extensive simulations in $\mathrm{C}$ and MATLAB to validate the performance of the proposed channel allocation technique. We use a grid size of $100 \times 100$ square units and to emulate primary behavior, we use the model and the range of values from the real-world spectrum data archive of RWTH Mobnets [5]. We consider a basic path-loss model and assume 50-400 channels of bandwidth $2 \mathrm{MHz}-250 \mathrm{kHz}$ each.

Fig. 4 shows the estimated received signal strength from primaries (noise from primary incumbent) for a typical IEEE 802.22 cell at a particular distance from primary transmitter. The transmitter located at $(30,45)$ is assumed to transmit at $1 \mathrm{~mW}$. This power profile is estimated by an IEEE 802.22 BS which resides in the middle of the cell. All the points in the region can be thought of as potential CPE locations. Through such RSS estimation, the BS predicts channel occupancy in its cell and computes the free channels at a particular CPE.

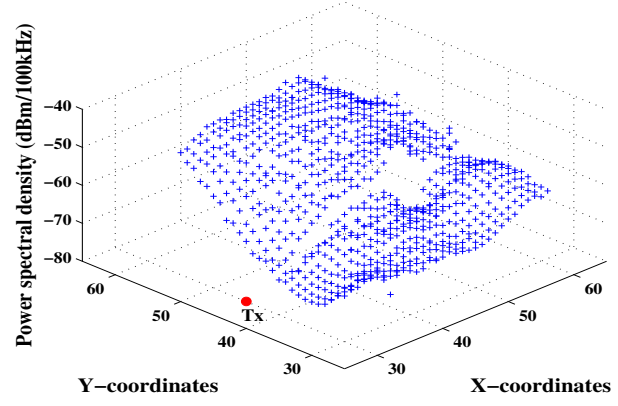

Fig. 4. Estimated RSS for an IEEE 802.22 cell 


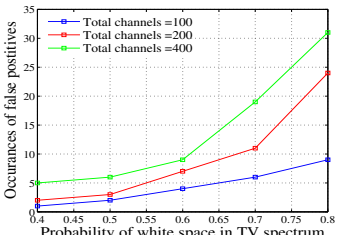

(a) Nature of occurances of false positives in estimation

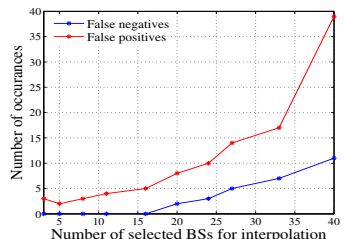
varying number of selected BSs (b) Accuracy of estimation with

Fig. 5. Accuracy of spectrum usage estimation

In Fig. 5(a), we show how occurrences of false positives in estimation vary with the probability of getting white space. We vary the total number of available channels (bandwidth of each channel) in order to determine its effect on the occurrences of false positives. We observe that as there are more channels available for the secondary nodes to use, the conservative approach of the estimation technique becomes more predominant. However, we see that even with $80 \%$ probability of getting free channels the ratio of instances of false positives to total number of channels is less than 0.1 .

Fig. 5(b) depicts the accuracy of the estimation technique in terms of instances of both false positives and false negatives with varying number of neighboring BSs consulted for the interpolation purpose. We see a sharp increase in both instances when more than $15 \mathrm{BSs}$ are selected. This finding is in accordance with Shepard's [7] claim about the existence of upper and lower bounds on the number of data-points to be used for most accurate estimation. This upper bound on number of selected BSs helps us to choose optimal search radius depending on the density and topology of an IEEE 802.22 cell.

Figures 6(a) and 6(b) collectively portray how the available channels in the spectrum are utilized by the proposed allocation technique. In Fig. 6(a), a line with $45^{\circ}$ slope signifies $100 \%$ utilization which occurs when there are more channels available to allocate to the CPEs. However even with 50 channels with probability of 0.6 , we see that 17 free channels are allocated resulting $57 \%(17 /[50 \times 0.6])$ utilization.

Nature of free channel utilization with varying number of available channels in the spectrum is better understood in Fig. 6(b) where we vary total number of channels in the spectrum and probability of getting white space thereby indirectly varying number of free channels to utilize. We observe that even with a very low probability of white space 0.4 (with respect to TV spectrum), the proposed allocation scheme ensures more than $50 \%$ utilization of free spectrum in most occasions. The total number of allocable CPEs are kept at 255 for all the scenarios above which is the maximum number of CPEs that an IEEE 802.22 cell can have [1].

In Fig. 7, we show the efficiency of the proposed allocation technique by calculating the number of CPEs being allocated a pair of uplink and downlink channels. We observe that with 400 channels and a probability of getting white space of 0.8 , we allocate 106 out of possible 160 CPEs.

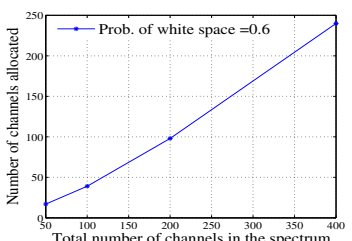

(a) Number of channel allocated from total available channels

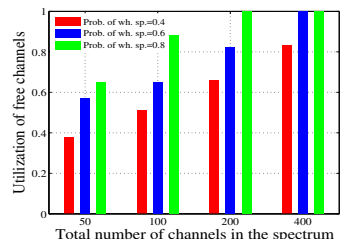

(b) Utilization of available chanwhite space nels for different probailities of

Fig. 6. Utilization of available channels in the spectrum

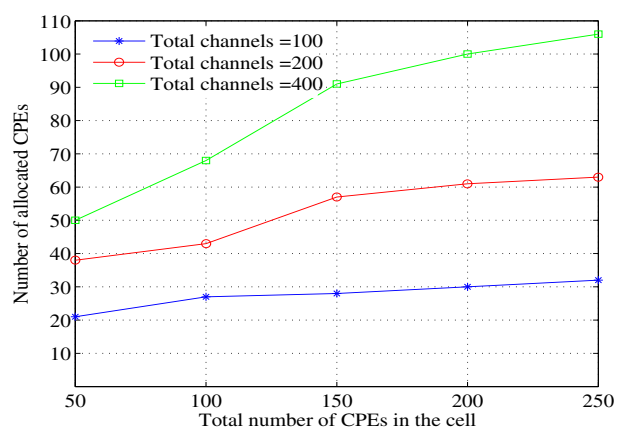

Fig. 7. Number of allocated CPEs for different number of channels

\section{CONCLUSIONS}

In this paper, we proposed a spectrum usage estimation technique and channel allocation scheme for IEEE 802.22 networks. We modified Shepard's interpolation technique for a BS to fuse information gathered from other neighboring BSs and predict the spectrum usage scenario at any location in its cell. We showed how a BS can make wise uplink and downlink channel allocations based on channel occupancy vector. We conducted simulation experiments to demonstate the effciency of the proposed techniques in terms of accuracy of estimation, utilization of free spectrum, and effectiveness of the allocation technique.

\section{REFERENCES}

[1] IEEE P802.22/D1.0 Draft Standard for Wireless Regional Area Networks Part 22: Cognitive Wireless RAN Medium Access Control (MAC) and Physical Layer (PHY) Specifications: Policies and Procedures for Operation in the TV Bands, April 2008

[2] IEEE 802.22, Working Group on Wireless Regional Area Networks (WRAN), http://grouper.ieee.org/ groups /802/22

[3] Akyildiz, I. F.; Lee, W. Y.; Vuran, M.C.; Mohanty, S.; , "NeXt Generation/Dynamic Spectrum Access/Cognitive RadioWireless Networks: A Survey, "Elseview Journal of Computer Networks, Vol. 50, pp. 2127-2159, September 2006

[4] Lazos, L.; Sisi Liu; Krunz, M.; , "Spectrum Opportunity-Based Control Channel Assignment in Cognitive Radio Networks," Sensor, Mesh and Ad Hoc Communications and Networks, 2009. SECON '09. 6th Annual IEEE Communications Society Conference on , pp. 135-143, 22-26 June 2009

[5] Riihijarvi, J.; Mahonen, P.; Wellens, M.; Gordziel, M.; , "Characterization and modelling of spectrum for dynamic spectrum access with spatial statistics and random fields," Personal, Indoor and Mobile Radio Communications, 2008. PIMRC 2008. IEEE 19th International Symposium on , pp.1-6, 15-18 Sept. 2008

[6] Sengupta, S.; Chandramouli, R.; Brahma, S.; Chatterjee, M.; , "A game theoretic framework for distributed self-coexistence among IEEE 802.22 networks," Global Telecommunications Conference, 2008. IEEE GLOBECOM 2008. IEEE , pp. 1-6, Nov. 30 2008-Dec. 42008

[7] Shepard, D.; , "A Two-Dimensional Interpolation Function for Irregularly-Spaced Data," ACM National Conference, 1968

[8] Tachwali, Y.; Barnes, W.J.; Basma, F.; Refai, H.H.; , "The Feasibility of a Fast Fourier Sampling Technique for Wireless Microphone Detection in IEEE 802.22 Air Interface," INFOCOM IEEE Conference on Computer Communications Workshops , 2010 , pp. 1-5, 15-19 March 2010 